\title{
CONVECTION AND ITS STABILITY IN THE EQUATORIAL REGIONS OF THE CONVECTION ZONE
}

\author{
A.V. KLYACHKIN \\ Ioffe Physical-Technical Institute \\ Academy of Sciences \\ Ul. Politechnicheskaya 26 \\ 194021, Leningrad, USSR
}

The problem of the existence, evolution, and stability of spatial structures in convection is of considerable importance to astrophysics as well as to geophysical hydrodynamics. The Boussinesq approximation will be used because the considered motions in stars are sufficiently slow. The system of hydrodynamic equations describing convection in a rotating inhomogeneous medium has the form:

$$
\begin{aligned}
D_{t} \mathbf{U}+2 \Omega \times \mathbf{U} & =-\rho_{m}^{-1} \nabla P+\left(\beta_{c} C-\beta_{T} T\right) \mathbf{g}+\nu_{m} \Delta \mathbf{U} \\
D_{t} T+\mathbf{U} \cdot\left(\nabla T_{b}-\nabla T_{a d}\right) & =\chi_{m} \Delta T \\
D_{t} C+\mathbf{U} \cdot \nabla C_{b} & =\mathcal{D}_{m} \Delta C \\
\operatorname{div} \mathbf{U}=0 &
\end{aligned}
$$

Here $D_{t}$ is the total time derivative, $\mathrm{U}$ the velocity, $P, T$, and $C$ the deviations of the pressure, temperature, and helium abundance (by mass) from the basic equilibrium values, $\rho_{m}, \nu_{m}, \chi_{m}$, and $\mathcal{D}_{m}$ the values averaged over the considered layer of the density, viscosity, thermal and helium diffusivities, $\beta_{T}$ and $\beta_{c}$ the averaged coefficients of the thermal and helium expansions, $\mathbf{g}$ and $\boldsymbol{\Omega}$ the gravitational acceleration and angular velocity, $\nabla T_{b}$ and $\nabla C_{b}$ the values of the basic equilibrium temperature and helium gradients, and $\nabla T_{a d}$ the adiabatic temperature gradient.

An analysis of finite-amplitude convection in a rotating non-uniform star has been performed by Dolginov and Klyachkin $(1987,1988,1989)$ using bifurcation theory methods applied to Eq. (1). It was shown that for a wide range of physical parameters such as rotation rate and degree of chemical inhomogeneity $\left(\nabla C_{b}\right)$ convection arises when the initial temperature gradient exceeds a critical value (supercritical bifurcation). Furthermore the existence of convective motions for values of the basic temperature gradient less than the critical value (subcritical bifurcation) was found to be possible in the case of slow rotation and a sufficiently large downwards directed helium gradient. In both cases the convection occurs as waves in the form of banana cells elongated along the meridian with an equatorial amplitude maximum. These waves are propagating only azimuthally, both along and opposite to the direction of rotation. The presence of a chemical inhomogeneity in the considered 
layer is very important for the physics of the convection processes. It leads not only to a change of the critical temperature gradient, but also to the appearance of slow gyroscopic waves (of Rossby type), with two double-diffusion modes determined by the competition between the thermal and helium diffusions.

It has however not been known whether the spatial structures are stable and how their evolution may be influenced by nonlinear interaction and energy transfer between the different generated modes. For a more detailed analysis of convection in the weakly nonlinear regime we will therefore use the method of amplitude equation, which is based on multiple scalings in space and time. As the propagation of waves is one-dimensional, only the azimuthal coordinate $\varphi$ is scaled. Let us choose the scaling

$$
\tau_{1}=\epsilon t, \quad \tau_{2}=\epsilon^{2} t, \quad \varphi_{1}=\epsilon \varphi, \varphi_{2}=\epsilon^{2} \varphi,
$$

where $\epsilon$ is a measure of supercriticality defined by $R_{T}=R_{0}+\epsilon^{2} R_{2}$. Here $R_{T}$ is the thermal Rayleigh number, which is proportional to the basic temperature gradient, while $R_{0}$ is the critical value of $R_{T}$, as obtained with a linear approximation. We seek a solution of Eq. (1) in the form of an expansion in $\epsilon$, which is a measure of the convection amplitude.

$$
(V, W, T, C)=\sum_{n=1} \epsilon^{n}\left(V_{n}, W_{n}, T_{n}, C_{n}\right),
$$

where $V$ and $W$ are the poloidal and toroidal velocity potentials $\left(\mathbf{U}=\left[\operatorname{curl}^{2} \mathbf{r} V+\operatorname{curl} \mathbf{r} W\right] r_{0}^{-1}\right.$, with $r_{0}=R / h$ being the inner radius of the considered spherical shell in units of its depth). According to DiPrima et al. (1971), the multi-scaling method can be used for a Hopf bifurcation in the bounded spatial domain, if the wave number spectrum is sufficiently dense: $\Delta k \lesssim \epsilon^{2}<1$. For a spherical shell, $\Delta k=r_{0}^{-1}$, and therefore the amplitude equation can correctly describe the convection process in a thin $\left(r_{0}>1\right)$ spherical layer. Physically it means that in spite of small supercriticality a great number of different spatial harmonics take part in the nonlinear interaction. As a result of the condition that Eq. system (1) has solutions in the $O\left(\epsilon^{3}\right)$ approximation (for details, see Klyachkin, 1989), the amplitude equation follows:

$$
\partial A / \partial \tau_{2}=\alpha A-\gamma|A|^{2} A+\beta \partial^{2} A / \partial x_{1}^{2}
$$

It is a complex $\left(\beta=\beta_{r}+i \beta_{I}, \gamma=\gamma_{r}+i \gamma_{I}\right)$, one-dimensional Ginzburg-Landau equation. Here $A=A\left(\tau_{2}, x_{1}, x_{2}\right)$ is a slowly varying complex amplitude, $x_{1}=\epsilon\left(\varphi-c_{g} t\right), x_{2}=\epsilon^{2}\left(\varphi-c_{g} t\right)$, and $c_{g}$ is the group speed of the most unstable mode. The velocity, temperature, and helium concentration depend both on the slow variables (reflecting the mutual interdependence of the modes) and on the rapid variables (reflecting the spatial and temporal dependence of the most unstable mode). For example

$$
T_{1}=\operatorname{Re} \hat{T}_{1} A\left(\tau_{2}, x_{1}, x_{2}\right) P_{\ell}^{\ell}(\cos \theta) \exp [i(\ell \varphi+\omega t)] \sin \pi\left(r-r_{0}\right),
$$

where $\hat{T}_{1}$ is a constant, chosen from the normalization condition. The coefficients $\alpha, \beta$, and $\gamma$ are uniquely connected with the controlling parameters describing the rotation, degree of chemical inhomogeneity of the medium, and its physical characteristics: viscosity, thermal and helium diffusivities. These expressions for $\alpha, \beta$, and $\gamma$ have been given in Klyachkin (1989). The obvious solutions of Eq. (2) are monochromatic, finite-amplitude rotating waves. It is known that chaotic solutions of Eq. (2) are possible. The condition for such solutions 
not to appear is also the condition for asymptotically reaching the monochromatic regime (Doering et al., 1988), as well as the condition for modulational stability of the spatially homogeneous solutions $\left(A=A\left(\tau_{2}\right)\right)$. This condition has the form $\beta_{r} \gamma_{r}+\beta_{I} \gamma_{I}>0$. It is satisfied for given values of $\alpha, \beta$, and $\gamma$ when rotation is not very rapid, and in the parameter domain corresponding to supercritical bifurcation in convection. For such parameter values convective motions appearing in the form of slow gyroscopic waves with retrograde propagation represent stable solutions. It is thus not surprising that gyroscopic waves have been found in numerical treatments of convection in a rotating, chemically homogeneous spherical shell in the mean-field approximation (Durney, 1970).

In the domain where subcritical bifurcation takes place, the coefficient $\gamma_{r}<0$, and according to Doering et al. (1988) an explosive growth of the solution in a finite time is possible in this case. Phenomenologically this growth can be limited by adding a term with a higher degree of nonlinearity (Landau and Lifshits, 1986):

$$
\partial A / \partial \tau_{2}=\alpha A-\gamma|A|^{2} A-\mu|A|^{4} A+\beta \partial^{2} A / \partial x_{1}^{2} .
$$

The evident solutions of Eq. (3), finite-amplitude waves, can possess both small and large amplitudes when the wave number is fixed. The analysis shows that all small-amplitude solutions are modulationally unstable. The spatially homogeneous $\left(A=A\left(\tau_{2}\right)\right)$ large-amplitude solutions are modulationally stable if

$$
\left(\beta_{r} \mu_{r}+\beta_{I} \mu_{I}\right)\left(\gamma_{r}^{2}+4 \mu_{r} \alpha\right)^{1 / 2}+\beta_{I}\left(\gamma_{I} \mu_{r}-\gamma_{r} \mu_{I}\right)>0 .
$$

For spatially inhomogeneous solutions $A=a \exp \left[i\left(k x_{1}+\omega \tau_{2}\right)\right]$, the neutral curves of modulational stability relative to perturbations with wave number $\tilde{k}$ have the form (for details, see Klyachkin, 1989)

$$
\begin{aligned}
4 k^{2} \beta_{r}^{2}= & {\left[|\beta|^{2} \tilde{k}^{2}+2 a^{2}\left(\beta_{r} \gamma_{r}+\beta_{I} \gamma_{I}\right)+4 a^{4}\left(\beta_{r} \mu_{r}+\beta_{I} \mu_{I}\right)\right] } \\
& \left\{1+\left[\beta_{I} \tilde{k}^{2}+\gamma_{I} a^{2}+2 \mu_{I} a^{4}\right]^{2}\left[\beta_{r} \tilde{k}^{2}+\gamma_{r} a^{2}+2 \mu_{r} a^{4}\right]^{-2}\right\}^{-1} .
\end{aligned}
$$

Here

$$
a^{2}=\left[-\gamma_{r} \pm \sqrt{\gamma_{r}^{2}+4 \mu_{r}\left(\alpha-\beta_{r} k^{2}\right)}\right]\left(2 \mu_{r}\right)^{-1}
$$

Criterion (4) is identical for $\mu=0$ with the analogous one obtained by Doering et al. (1988).

Let us finally point out that Eqs. (2) and (3) do not describe the processes taking place near the multiple bifurcation line of codimension 2 or the multiple bifurcation point of codimension 3, which occur when a definite relation between rotation, external heating, and degree of chemical inhomogeneity exists.

The author is grateful to Prof. A.Z. Dolginov for fruitful discussions and valuable comments.

\section{References}

DiPrima, R.C., Eckhaus, W., and Segel, L.A. (1971) 'Non-linear wave-number interaction in near-critical two-dimensional flows', $J$. Fluid Mech. 49, 705-744. 
Doering, C.R., Gibbon, J.D., Holm D.D., and Nicolaenko, B. (1988) 'Low-dimensional behaviour in the complex Ginzburg-Landau equation', Nonlinearity 1, 279-309.

Dolginov, A.Z. and Klyachkin, A.V. (1987) 'The influence of the helium abundance on the convection threshold in surface layers of rotating stars', Piz'ma v Astronomicheskii Zhurnal 13, 764-772.

Dolginov, A.Z. and Klyachkin, A.V. (1988) 'Influence of the chemical composition on the convection, meridional circulation and differential stellar rotation', in Yu.V. Glagolevsky and J.M. Kopylov (eds.), Magnetic Stars; "Nauka", Leningrad, pp. 264-280.

Dolginov, A.Z. and Klyachkin, A.V. (1989) 'Convection in the equatorial region of a rotating chemically nonuniform star', Piz'ma v Astronomicheskii Zhurnal 15, 33-41.

Durney, B. (1970) 'Nonaxisymmetrical convection in a rotating spherical shell', Astrophys. J. 161, 353-367.

Klyachkin, A.V. (1989) 'The modulation of the autowave convective structures on the rotating chemically nonuniform stars', Preprint No. 1338, Physical-Technical Ioffe Institute, Leningrad.

Landau, L.D. and Lifshits, E.M. (1986) Gidrodinamika, "Nauka", Moscow. 